\title{
Modeling Minority-Carrier Lifetime Techniques That Use Transient Excess-Carrier Decay
}

Conference Paper NREL/CP-520-42569

May 2008

\section{Preprint}

S.W. Johnston

National Renewable Energy Laboratory

G.M. Berman

University of Colorado - Boulder

R.K. Ahrenkiel

Colorado School of Mines

Presented at the 33rd IEEE Photovoltaic Specialists Conference San Diego, California

May 11-16, 2008

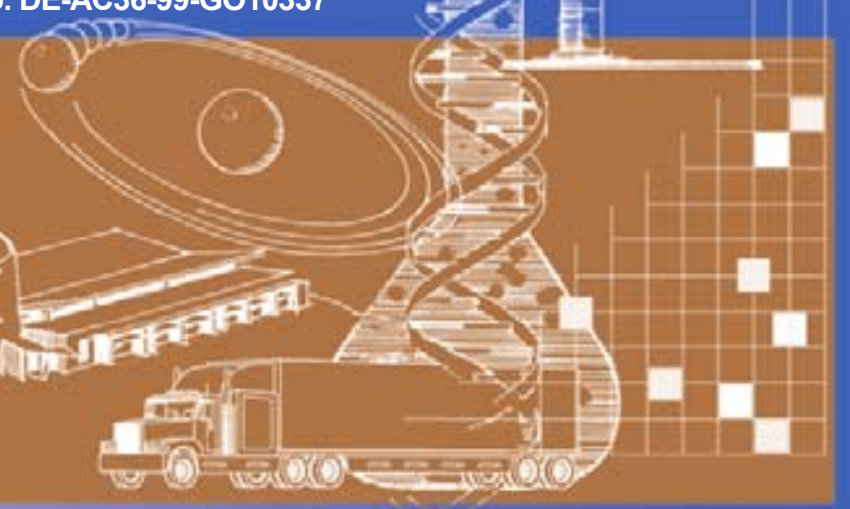




\section{NOTICE}

The submitted manuscript has been offered by an employee of the Midwest Research Institute (MRI), a contractor of the US Government under Contract No. DE-AC36-99G010337. Accordingly, the US Government and MRI retain a nonexclusive royalty-free license to publish or reproduce the published form of this contribution, or allow others to do so, for US Government purposes.

This report was prepared as an account of work sponsored by an agency of the United States government. Neither the United States government nor any agency thereof, nor any of their employees, makes any warranty, express or implied, or assumes any legal liability or responsibility for the accuracy, completeness, or usefulness of any information, apparatus, product, or process disclosed, or represents that its use would not infringe privately owned rights. Reference herein to any specific commercial product, process, or service by trade name, trademark, manufacturer, or otherwise does not necessarily constitute or imply its endorsement, recommendation, or favoring by the United States government or any agency thereof. The views and opinions of authors expressed herein do not necessarily state or reflect those of the United States government or any agency thereof.

Available electronically at http://www.osti.gov/bridge

Available for a processing fee to U.S. Department of Energy and its contractors, in paper, from:

U.S. Department of Energy

Office of Scientific and Technical Information

P.O. Box 62

Oak Ridge, TN 37831-0062

phone: 865.576 .8401

fax: 865.576 .5728

email: mailto:reports@adonis.osti.gov

Available for sale to the public, in paper, from:

U.S. Department of Commerce

National Technical Information Service

5285 Port Royal Road

Springfield, VA 22161

phone: 800.553 .6847

fax: 703.605.6900

email: orders@ntis.fedworld.gov

online ordering: http://www.ntis.gov/ordering.htm 


\title{
MODELING MINORITY-CARRIER LIFETIME TECHNIQUES THAT USE TRANSIENT EXCESS-CARRIER DECAY
}

\author{
Steven W. Johnston ${ }^{1}$, Gregory M. Berman ${ }^{1,2}$, and Richard K. Ahrenkiel ${ }^{1,3}$ \\ ${ }^{1}$ National Renewable Energy Laboratory, Golden, CO 80401 \\ ${ }^{2}$ Optical Science \& Engineering Program, University of Colorado, Boulder, CO 80304 \\ ${ }^{3}$ Colorado School of Mines, Golden, CO 80401
}

\begin{abstract}
Details of the operation of a photoconductive decay technique called resonant-coupled photoconductive decay are revealed using modeling and circuit simulation. The technique is shown to have a good linear response over its measurement range. Experimentally measured sensitivity and linear response compare well to microwave reflection at low injection levels. We also measure the excess-carrier decay rate by an infrared free-carrier transient absorption technique and show comparable high injection level lifetimes.
\end{abstract}

\section{INTRODUCTION}

Lifetime spectroscopy is a valuable tool for the characterization of photovoltaic materials. Measured lifetime values are inherently dependent on the defect and impurity densities present in the material. Injection level dependencies of the recombination rates further characterize the material and possibly provide information for the identification of specific impurities. Injection level dependent measurements are also applicable when measuring lifetime with respect to the material-doping level or the device-operating level, such as 1-sun intensity for typical solar cells. The technique for measuring injection-level dependent lifetime therefore needs to have linear response and high sensitivity for measuring over large ranges of excited excess-carrier density.

Lifetime may be measured using contactless, transient photoconductive decay techniques such as microwave reflection photoconductive decay ( $\mu P C D)$ or resonant-coupled photoconductive decay (RCPCD). For $\mu \mathrm{PCD}$ (few to tens of $\mathrm{GHz}$ frequencies), the sample is coupled to the end of a microwave waveguide or antenna structure, and changes in reflected microwave power are sensed as short light pulses generate photoconductivity [1-6]. Similarly, RCPCD ( 500 MHz) uses reflected power from the sample, which is coupled to a small coil, or other antenna structure [7-9]. For $\mu P C D$, the sample is impedance matched to the waveguide by using an $E$ and $\mathrm{H}$ plane tuner [3], which are sliding shorts in each plane of the waveguide. For RCPCD, the antenna with coupled sample can be impedance matched by adjusting a variable capacitor, frequency, and/or mutual coupling to the sample [9]. For each case, impedance matching is performed without the pulsed light source creating excess carriers, and the circuit's zero signal, or null, corresponds to no reflected power from the sample. Excess carriers are created using a pulsed light source. For each pulse, changes in sample conductivity disrupt the impedancematched circuit balance, resulting in reflected power that is detected and recorded using an oscilloscope.

\section{MODELING RCPCD}

RCPCD is modeled by forming an equivalent circuit. The most variable element, and difficult to model, is the coupling of the antenna structure, in this case a multi-turn coil, to the sample. The samples can vary greatly in size, shape, thickness, and doping, or conductivity. The circuitry, therefore, needs tunable parameters to account for the varying sample conditions. These are the coupling distance, operating frequency, and a tunable capacitor. We begin with modeling the coupling of the sample to a coil antenna. Figure 1 shows a photo of the coil coupled to a small piece of semiconductor. The impedance of the coil (coupled to the sample) is measured by directly placing the coil on an impedance analyzer. The change in impedance due to photoconductance is measured by shining light on the sample. A sample with $\sim 100 \mathrm{ohm}-\mathrm{cm}$ resistivity was used so that the relatively weak light source could sufficiently increase the conductivity. Figure 2 shows the changing impedance as a function of increasing light intensity for a sample very near the coil and then spaced $2 \mathrm{~mm}$ and $4 \mathrm{~mm}$ away from the coil. The change in real impedance, or resistance, is plotted against the change in inductance from positive imaginary impedance.

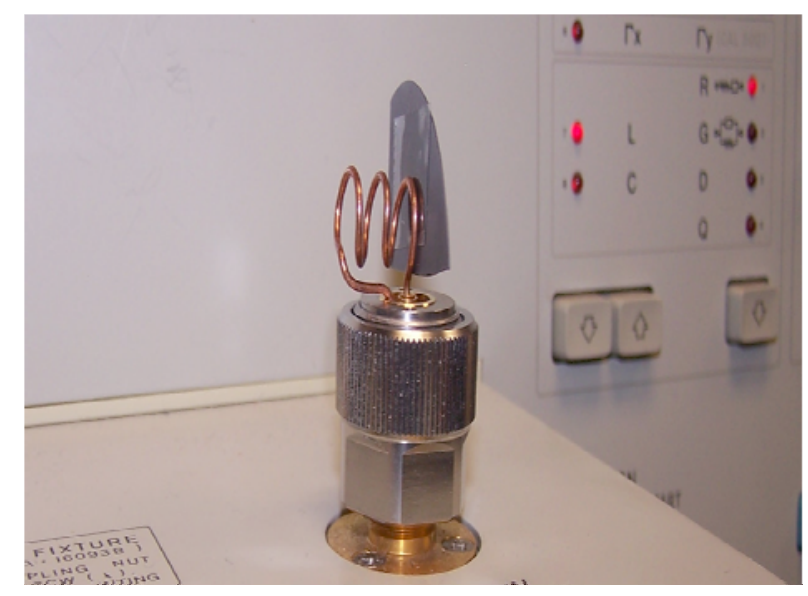

Figure 1. Photograph of sample (wafer piece) coupled to coil antenna and mounted on an impedance analyzer. 


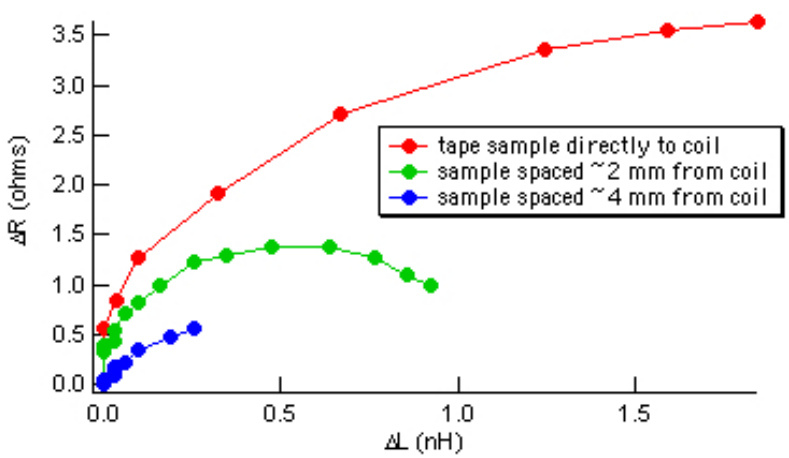

Figure 2. Impedance analyzer data for increasing photoconductivity and varying sample coupling.

The coupling of the sample to the coil antenna can be thought of as a transformer, where the coil is a single turn secondary coupled to the primary coil by a mutual conductance. This equivalent circuit is shown in Fig. 3.

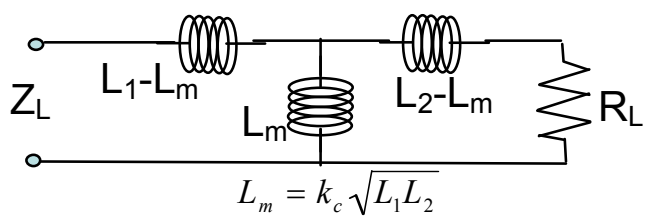

Figure 3. Equivalent circuit for sample coupled to coil using transformer model.

The sample's conductivity and changing photoconductivity are represented by the secondary winding's resistance, $R_{L}$. The mutual inductance, $L_{m}$, depends upon the coupling coefficient, $\mathrm{k}_{\mathrm{c}}$. Figure 4 shows that values of inductance and coupling coefficient can be chosen that result in similarly shaped responses of changing resistance versus inductance.

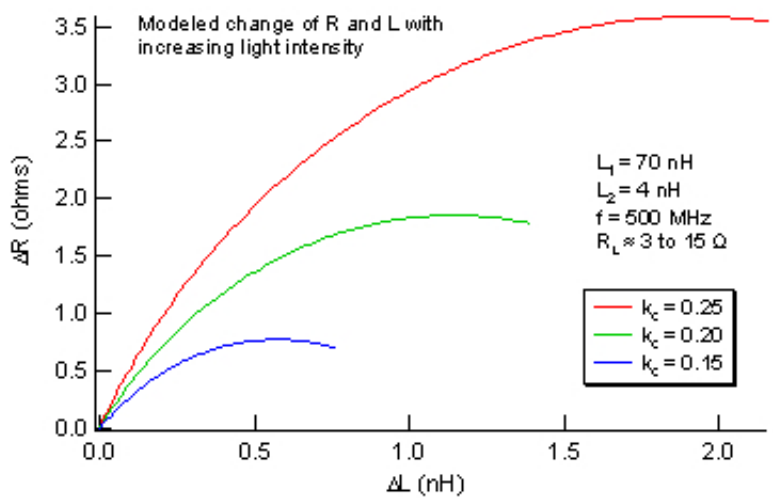

Figure 4. Modeling results of transformer equivalent circuit for various coupling distances.

The transformer model is a simplistic equivalent circuit with many fitting parameters. We have also used Ansoft's high frequency simulation solver (HFSS) for three-dimensional solution of electromagnetic fields. The model is drawn as shown in Fig. 5. The software creates a mesh of points and solves numerical electromagnetic equations by iterations until converging to a solution.

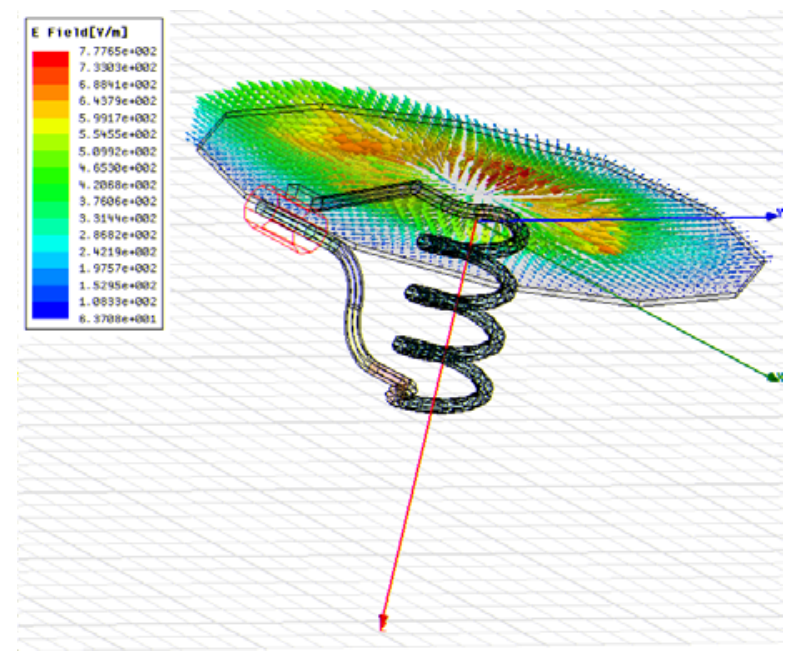

Figure 5. HFSS model for a sample coupled to a coil antenna. The electric field values are illustrated by color and increase from blue to red.

The sample was chosen to have an initial resistivity of $100 \mathrm{ohm}-\mathrm{cm}$. Impedance data was calculated as the resistivity was reduced to $\sim 2 \mathrm{ohm}-\mathrm{cm}$. These data were then offset so that the initial resistivity corresponds to values of zero for $\Delta R$ and $\Delta X$. As shown in Fig. 6 , the response of $\Delta R$ versus $\Delta X$ is similar to the measured values and those given by the transformer model.

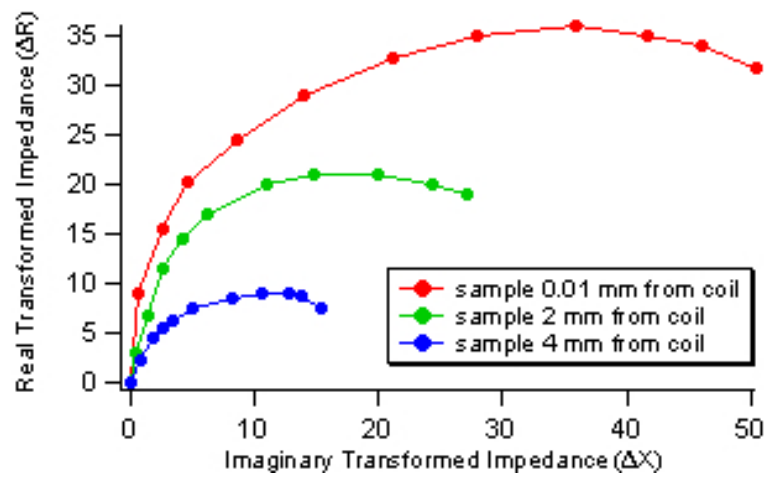

Figure 6. Response of changing resistance, $\Delta R$, and changing reactance, $\Delta \mathrm{X}$, as a function of increasing conductivity using the Ansoft HFSS modeling software.

The coil antenna is connected to a coaxial cable. The length of the cable is important as it assists in determining the tuning frequency. The coil is soldered to $50 \Omega$, semirigid, coaxial cable that is approximately $150 \mathrm{~mm}$ in length. The circuit's impedance as a function of frequency are measured using the impedance analyzer, and the results are shown in Fig. 7(a). 

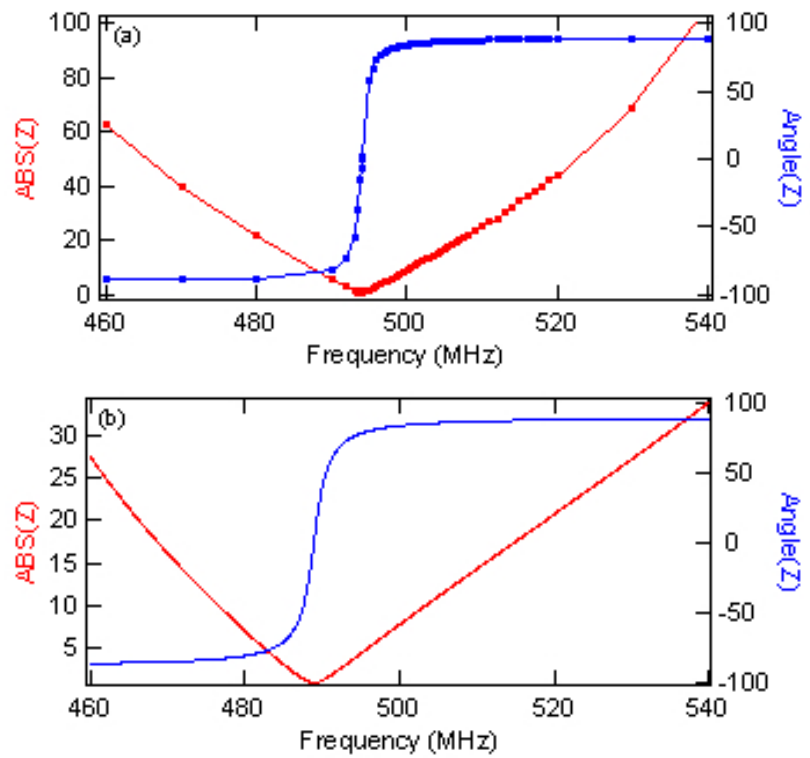

Figure 7. (a) Impedance analyzer data for the coil on a length of coaxial cable. (b) Modeled circuit for coil on length of coaxial cable.

The length of coaxial cable connected to the coil can also be modeled. The coil is modeled using the transformer model from before, and the coaxial cable is modeled using the transmission line formula of Equation 1.

$$
Z_{\text {trans }}=Z_{o} \frac{Z_{L}-i Z_{o} \tan (\beta z)}{Z_{o}-i Z_{L} \tan (\beta z)}
$$

$Z_{O}$ is $50 \Omega$, the characteristic impedance of the coaxial cable. $Z_{L}$ is the equivalent impedance of the coil and sample using the transformer circuit. $Z_{\text {trans }}$ is the impedance seen at the input of the coaxial cable, $z$ is length, and $\beta=2 \pi / \lambda$, where $\lambda$ is wavelength. The modeling results shown in Fig. 7 (b) give similar impedance characteristics to those that were measured.

A variable capacitor is used to impedance match the coil, sample, and length of coaxial cable to the characteristic impedance of $50 \Omega$. The capacitor is enclosed in a metal-shielded box to reduce radiation and decrease losses. This air gap capacitor has low frequency values ranging from approximately 10 to $35 \mathrm{pF}$; however, the capacitor is used when it is near its self-resonance frequency. At these high frequencies, the capacitor is modeled by a distributed circuit analysis, which accounts for high-frequency behavior. [10] As shown in Fig. 8, the high-frequency modeling data of the capacitor fit the impedance analyzer measurement data very well.

Adding the capacitor to the input end of the coaxial cable leads to the configuration needed to create the impedance matching condition. The capacitor is in parallel; it bridges across from the inner conductor of the coaxial cable to outer conductor as shown in Fig. 9.

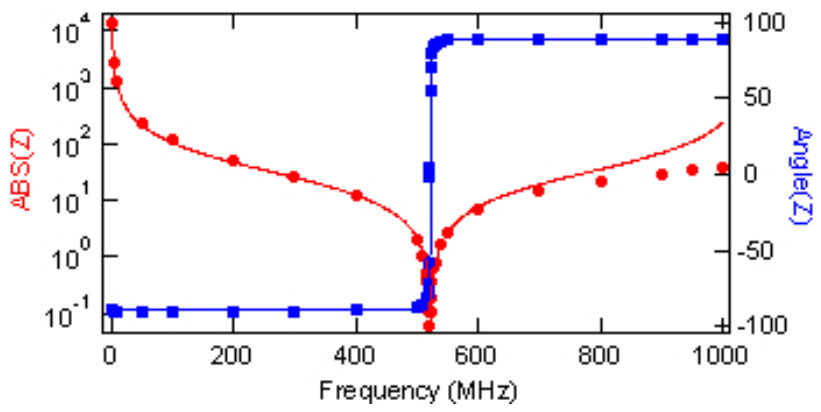

Figure 8. Measured data (markers) and modeled fit (lines) for a tunable capacitor set to low frequency value of $15 \mathrm{pF}$.

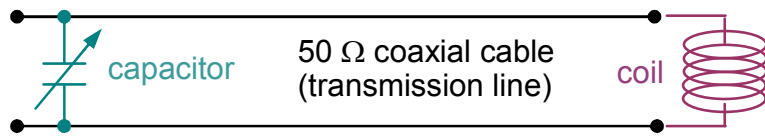

Figure 9. RCPCD partial circuit schematic.

The circuit as shown in Fig. 9 was connected and measured on the impedance analyzer. These results are shown in Fig. 10(a). Features relate to resonances as the coaxial cable varies between capacitive and inductive, and also the resonance of the capacitor near $500 \mathrm{MHz}$. This circuit is also modeled by combining the transformer model to represent the coil antenna and the sample, the transmission line equation to represent the coaxial cable length, and the high frequency capacitor model. The modeling results are shown in Fig. 10(b) and resemble the measured impedance values.
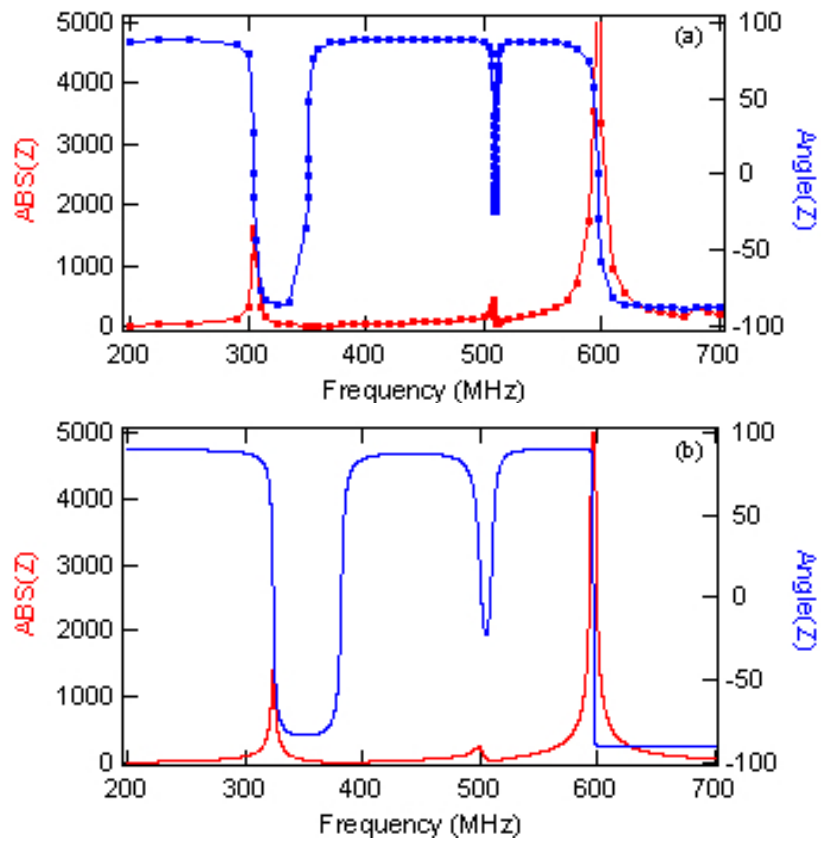

Figure 10. (a) Measured impedance values of circuit containing capacitor, coaxial cable, coil, and coupled sample. (b) Modeling of the same circuit. 
The most interesting feature to note is the resonance near $500 \mathrm{MHz}$ where the combination of coil impedance, coaxial cable length, and capacitance resonance lead to a circuit impedance resonance. This feature is shown expanded in Fig. 11.

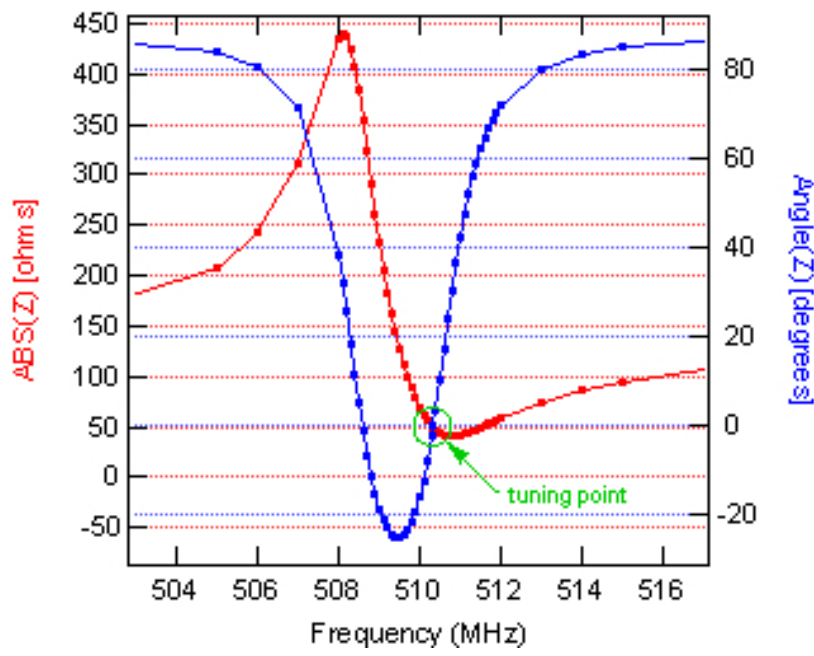

Figure. 11. Expanded view of the measured frequency response of the RCPCD circuit of Fig. 9.

Within the resonance feature near $500 \mathrm{MHz}$, there is a point where both the impedance magnitude is $50 \Omega$ and the impedance angle is $0^{\circ}$. Thus, the circuit looks perfectly impedance matched to $50 \Omega$ of real resistance at this particular tuning point. This tuning point depends upon the total impedance; thus, it depends upon the sample coupling and conductance. However, by adjusting two of three variables (variable capacitor and either frequency or sample coupling distance), the impedance matching point can be found, and this point serves as a baseline for the lifetime measurement.

For lifetime measurement, the circuit is initially tuned so that the input impedance looking into the capacitor is $50 \Omega$ real resistance. At this condition, power flows into this branch of the circuit, and no power is reflected back. Then, as a light pulse is absorbed in the sample, photoconductivity changes the sample's resistance, and the overall circuit's impedance. This impedance change results in the input impedance no longer looking like $50 \Omega$; thus, power is now reflected. A directional coupler is used as a "reflectometer" such that power is fed into the "out" port. Power then flows out of the "in" port and into the tuned antenna circuit branch. Impedance mismatch induced by pulsed light absorbed in the sample leads to reflected power that then re-enters the "in" port of the directional coupler. A portion of this power exits the directional coupler from the "coupled" port. A photo of the circuit is shown in Fig. 12. A circulator can also be used for this "reflectometer" component.

The RCPCD frequency operating point is where the system impedance is $50 \Omega$ of real resistance, and the

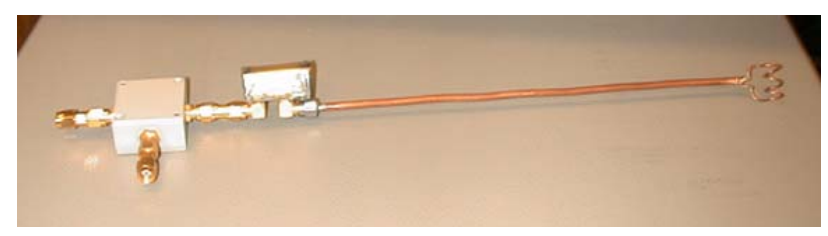

Figure 12. Photo of a branch of the RCPCD measurement circuit showing (from left to right) the directional coupler, shielded capacitor, length of coaxial cable, and coil antenna.

reflection coefficient, $\Gamma$, is nearly zero. Figure 13 shows details of the circuit branch impedance versus frequency. With no sample, or unloaded, the impedance matching frequency is near $434 \mathrm{MHz}$. This frequency point is shifted due to the measurement of a slightly different configuration that has a coaxial feedthrough and sample stage for sample positioning and laser light enclosure. The quality factor, $Q$, of the tuned impedance matching resonance is about 140 . Next, a sample is coupled to the coil antenna, and this now "loaded circuit" is retuned by adjusting the variable capacitor and the frequency. The operating frequency shifts to about $433 \mathrm{MHz}$, and the sample has induced more loss into the circuit that lowers the $Q$ to approximately 127 . Finally, light is directed onto the sample without retuning the circuit. This results in the curve labeled as "loaded \& light" which shows a slight shift in the curve again. To see the effect of the sample photoconductance, this area of the graph is expanded and shown in Fig. 14. Here, the initial reflection coefficient is seen to be 0.0007 , or nearly zero. With light, the reflection coefficient increases to 0.0356 at the circuit's operating frequency. Then, to complete the lifetime measurement circuitry, the reflected power is amplified, rectified with either a diode or mixer circuit, and displayed on an oscilloscope that is triggered in sync with the light pulses. Measurements can be averaged on the oscilloscope over tens to hundreds of light pulses to improve the signal-tonoise ratio.

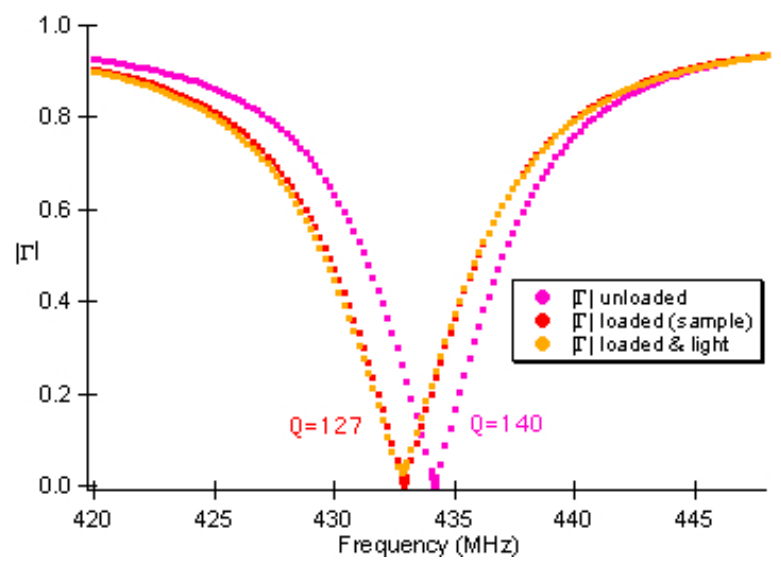

Figure 13. Frequency response of reflected power for circuit with no sample (unloaded), retuned circuit with sample (loaded), and then with sample illuminated (loaded \& light). 


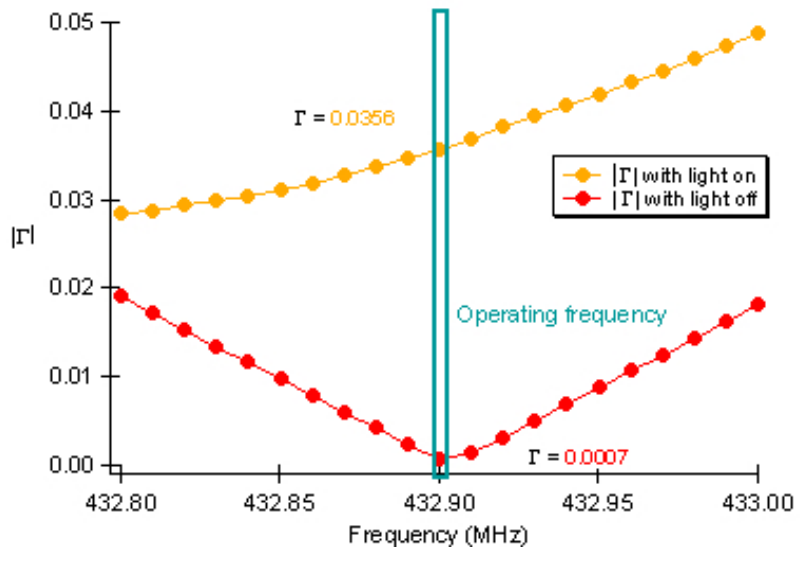

Figure 14. Expanded view of the operating frequency comparing reflected power of the circuit with sample in the dark to that of an illuminated sample.

For a linear response, the reflected power should vary with the sample photoconductance by a linearly proportional amount. As shown in Fig. 15(a), modeling of $\Gamma$ versus changing sample resistance implies a good linear relationship. As shown in Fig. 15(b), experimental measurement of $\Gamma$ versus photoconductivity confirms this relation. When $\Gamma \sim 0.05$, the output pulse of the amplifier
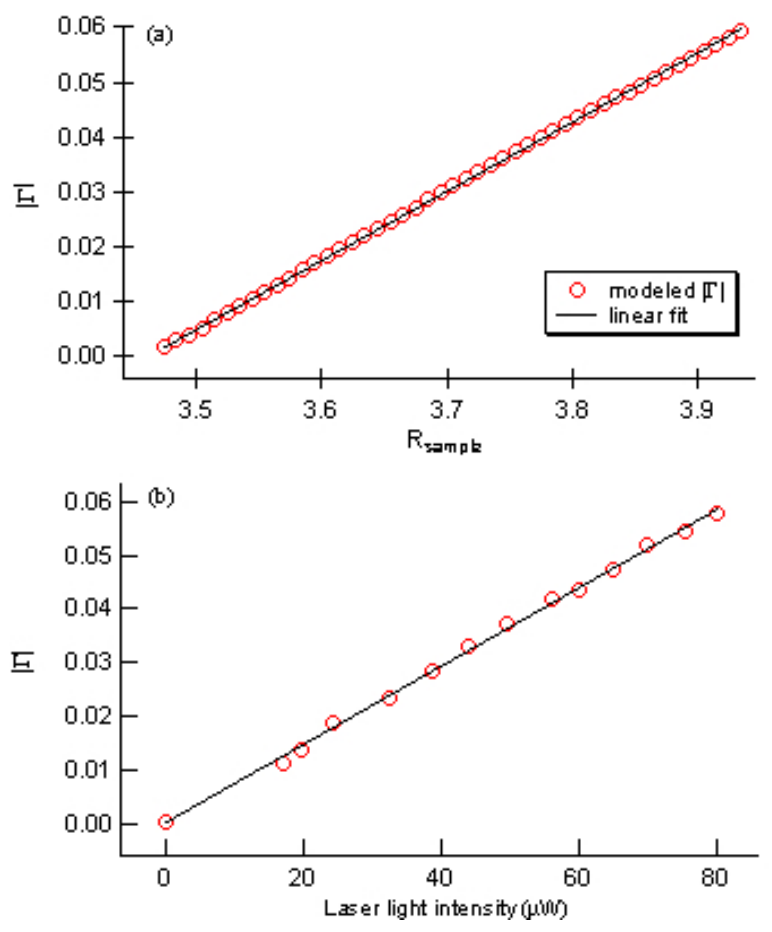

Figure 15. (a) Modeling of reflection coefficient, $\Gamma$, as a function of changing sample resistivity due to photoconductivity. (b) Measured reflection coefficient as a function of increasing light and photoconductivity of the sample. is nearly saturated, so measurements are made using $\Gamma<0.05$. In order to make measurements at higher injection levels, the sample can be coupled farther from the antenna to reduce the signal sensitivity.

\section{$\mu \mathrm{PCD}$}

The $\mu \mathrm{PCD}$ technique is well studied, and commercial measurement systems are available. Microwaves, typically in the 10 to $30 \mathrm{GHz}$ range, are reflected by the free carriers of the semiconductor, and the reflection coefficient is a function of carrier density. Pulsed light excites excess carriers, which modify the reflection coefficient of the microwave signal.

The reflection coefficient is a nonlinear function of sample resistance. Such nonlinearity may distort the transient time-resolved microwave reflectance signal. Small excess-carrier injection levels lead to small changes in microwave reflection that will accurately track changes in conductivity. But, at the highest conductivity ranges, the response will saturate, as the reflection coefficient approaches unity.

For our measurement setup, we use a frequency of $20 \mathrm{GHz}$ and corresponding waveguide components. The microwave power is supplied by a signal generator and then passes through an isolator before entering a directional coupler. After the directional coupler, the circuit contains an E-H plane tuner, which is used to minimize reflected power when the sample is positioned at the end of the open waveguide. Reflected power returns through the directional coupler and is coupled out to a $20 \mathrm{GHz}$ amplifier and finally a diode detector. The output is then amplified by a high bandwidth pulse amplifier and displayed on an oscilloscope. Once light pulses are exciting carriers in the sample, the $\mathrm{E}-\mathrm{H}$ plane tuner is adjusted to maximize the signal by finding the best offset bias for the diode detector.

\section{TRANSIENT FREE-CARRIER ABSORPTION}

Transient free-carrier absorption (FCA) is an optical technique based on measuring absorption changes of a transmitted infrared probe beam while excess carriers are excited by an absorbed light pulse [11]. The absorption coefficient is linearly dependent upon the density of free excess carriers; thus, the technique is capable of probing lifetime over a broad range of injection levels. Our measurement setup uses a $3.39 \mu \mathrm{m}$ wavelength $\mathrm{HeNe}$ laser as the probe beam and an InSb detector. The probe beam is incident on the sample at an angle of $\sim 25^{\circ}$.

\section{TECHNIQUE COMPARISON}

For all three measurements, we use a Nd:YAG pulsed laser with optical parametric oscillator tuned to $\sim 1100 \mathrm{~nm}$ wavelength as an excitation source for a silicon sample. The pulse width is $\sim 5 \mathrm{~ns}$, and the spot size is $\sim 1 \mathrm{~cm}$ in diameter. The laser pulses at $10 \mathrm{~Hz}$, and pulse-to-pulse averaging of the oscilloscope is used to improve the signal-to-noise ratio. 
For technique comparison, we chose a sample that could be measured on each technique using the same excitation laser and spot size. The sample is a doublesided polished silicon wafer with resistivity of $\sim 5 \mathrm{ohm}-\mathrm{cm}$ and $\sim 400 \mu \mathrm{m}$ thickness. At low injection levels, RCPCD and $\mu \mathrm{PCD}$ each show a lifetime near 13 to $14 \mu \mathrm{s}$, as shown in Figs. 16(a) and (b).
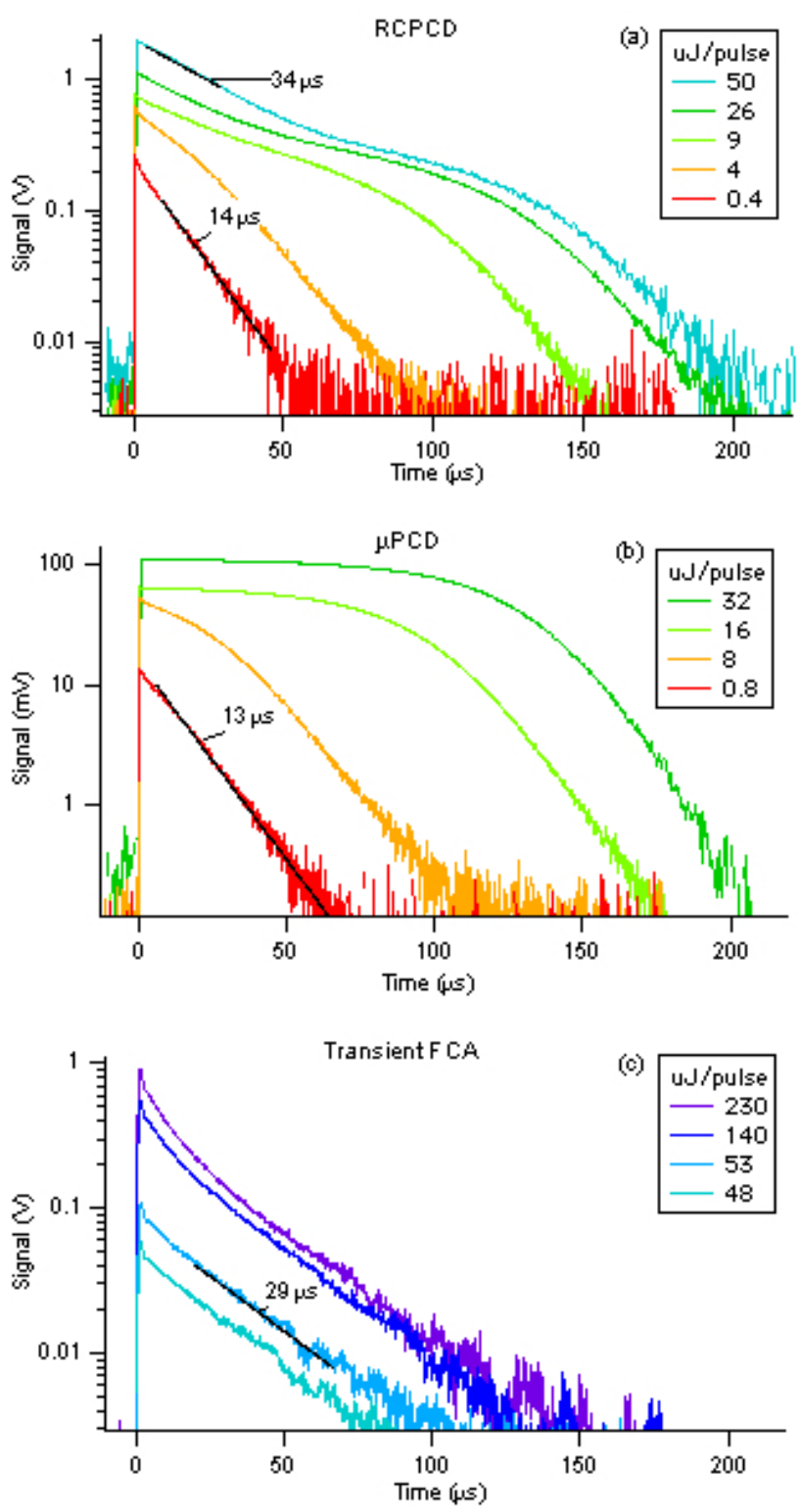

Figure 16. Transient photoconductive decay curves for a silicon sample measured at various injection levels for (a) RCPCD, (b) $\mu \mathrm{PCD}$, and (c) free-carrier absorption (FCA).

While the $\mu \mathrm{PCD}$ signal saturates at higher injection levels, the RCPCD signal has slope and does not saturate at the same injection levels. The RCPCD high injection decay rates are similar to those of transient FCA, which were also measured at high injection level. The transient FCA data are shown in Fig. 16(c). Unfortunately, our transient FCA technique so far does not have sufficient sensitivity to make measurements at lower injection levels.

\section{SUMMARY}

We have combined modeling and experimental results to illustrate the operation and injection-level dependent response of RCPCD. Simple circuit modeling has shown why we expect RCPCD to behave with a relatively linear response, even at high carrier injection levels. A measurement of RCPCD at varying injection levels was compared to measurements from $\mu P C D$ and transient FCA.

\section{ACKNOWLEDGEMENT}

This work was supported by the U.S. Department of Energy under Contract No. DE-AC36-99G010337 with the National Renewable Energy Laboratory.

\section{REFERENCES}

[1] S. Deb and B. R. Nag, "Measurement of Lifetime Carriers in Semiconductors through Microwave Reflection", J. Appl. Phys. 33, 1962, p. 1604.

[2] Y. Mada, "Nondestructive Method for Measuring the Spatial Distribution of Minority-Carrier Lifetime in Silicon Wafer", Japan. J. Appl. Phys. 18, 1979. pp. 2171-2172.

[3] A. Ohsawa et al., "Instrument Measuring Temperature Dependence of Minority-Carrier Lifetime Without Contact", Rev. Sci. Instrum. 54, 1983, pp. 210-212.

[4] M. Kunst and G. Beck, "The Study of Charge Carrier Kinetics in Semiconductors by Microwave Conductivity Measurements", J. Appl. Phys. 60, 1986, pp. 3558-3566.

[5] M. C. Chen, "Photoconductivity Lifetime Measurements on $\mathrm{HgCdTe}$ Using a Contactless Microwave Technique", J. Appl. Phys. 64, 1988, pp. 945-947.

[6] G. Q. Jiang et al., "Open-ended Coaxial-line Technique for the Measurement of the Microwave Dielectric Constant for Low-loss Solids and Liquids", Rev. Sci. Instrum. 64, 1993, pp. 1614-1621.

[7] T. Tiedje et al., "An RF Bridge Technique for Contactless Measurement of the Carrier Lifetime in Silicon Wafers", J. Appl. Phys. 54, 1983, pp. 2499-2503.

[8] E. Yablonovitch and T. J. Gmitter, "A Contactless Minority Lifetime Probe of Heterostructures, Surfaces, Interfaces and Bulk Wafers," Solid-State Elec. 35, 1992, pp. 261-267.

[9] R. K. Ahrenkiel and S. Johnston, "Contactless Measurement of Recombination Lifetime in Photovoltaic Materials", Solar Energy Materials \& Solar Cells 55, 1998, pp. 59-73.

[10] J. A. B. Faria, "Capacitor Modeling at High Frequencies--A Pedagogical Example of the Application of Distributed Circuit Analysis for Introductory Electromagnetics or Microwave Courses", IEEE Trans. on Edu. 35, 1992, pp. 214-216.

[11] J. Linnros, "Carrier Lifetime Measurements Using Free Carrier Absorption Transients. I. Principle and Injection Dependence", J. Appl. Phys. 84, 1998, pp. 275283. 


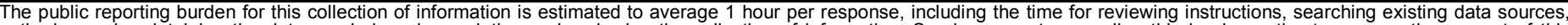

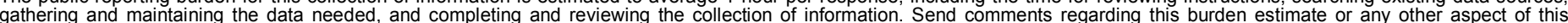

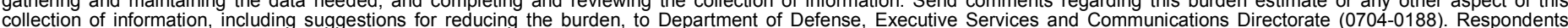

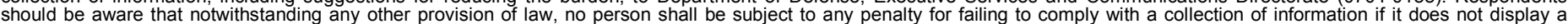

should be aware that notwithstanding

PLEASE DO NOT RETURN YOUR FORM TO THE ABOVE ORGANIZATION.

\section{REPORT DATE (DD-MM-YYYY) May 2008 \\ 2. REPORT TYPE \\ Conference Paper}

4. TITLE AND SUBTITLE

Modeling Minority-Carrier Lifetime Techniques That Use Transient

Excess-Carrier Decay: Preprint
3. DATES COVERED (From - To)

11-16 May 2008

5a. CONTRACT NUMBER

DE-AC36-99-G010337

5b. GRANT NUMBER

5c. PROGRAM ELEMENT NUMBER

5d. PROJECT NUMBER

NREL/CP-520-42569

5e. TASK NUMBER

PVA73101

5f. WORK UNIT NUMBER

\section{PERFORMING ORGANIZATION NAME(S) AND ADDRESS(ES)}

National Renewable Energy Laboratory, 1617 Cole Blvd., Golden, CO 80401-3393

Optical Science \& Engineering. Program, University of Colorado, Boulder,

Colorado 80304

Colorado School of Mines, Golden, Colorado 80401

9. SPONSORING/MONITORING AGENCY NAME(S) AND ADDRESS(ES)

10. SPONSOR/MONITOR'S ACRONYM(S) NREL

8. PERFORMING ORGANIZATION REPORT NUMBER

NREL/CP-520-42569

11. SPONSORING/MONITORING AGENCY REPORT NUMBER

12. DISTRIBUTION AVAILABILITY STATEMENT

National Technical Information Service

U.S. Department of Commerce

5285 Port Royal Road

Springfield, VA 22161

13. SUPPLEMENTARY NOTES

14. ABSTRACT (Maximum 200 Words)

Details of the operation of a photoconductive decay technique called resonant-coupled photoconductive decay are revealed using modeling and circuit simulation. The technique is shown to have a good linear response over its measurement range. Experimentally measured sensitivity and linear response compare well to microwave reflection at low injection levels. We also measure the excess-carrier decay rate by an infrared free-carrier transient absorption technique and show comparable high injection level lifetimes.

\section{SUBJECT TERMS}

PV; minority carrier; excess-carrier decay; transient absorption; photoconductive; circuit modeling;

16. SECURITY CLASSIFICATION OF:
\begin{tabular}{|l|l|l|}
\hline a. REPORT & b. ABSTRACT & c. THIS PAGE \\
Unclassified & Unclassified & Unclassified \\
& & \\
\hline
\end{tabular}

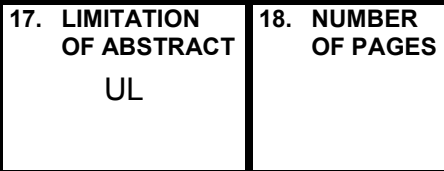

19a. NAME OF RESPONSIBLE PERSON

19b. TELEPHONE NUMBER (Include area code) 